\title{
In vivo and in vitro expression of the RASAL1 gene in human gastric adenocarcinoma and its clinicopathological significance
}

\author{
HONG CHEN $^{1}$, XIAO-WEI YANG ${ }^{1}$, HUI ZHANG ${ }^{1}$, QING YANG $^{1}$, ZHI WANG ${ }^{1}$, YANG LIU ${ }^{1}$, \\ FENG-LIN LU ${ }^{1}$, BAO-YI ZHOU ${ }^{2}$, CHENG-HUI QIU-XI ${ }^{2}$ and SHENG-LIN LU ${ }^{2}$ \\ ${ }^{1}$ Department of Gastroenterology, Zhongda Hospital, Southeast University, Nanjing 210009, Jiangsu; \\ ${ }^{2}$ Medical College, Southeast University, Nanjing 210009, Jiangsu, P.R. China
}

Received October 4, 2011; Accepted November 28, 2011

DOI: $10.3892 / \mathrm{ol} .2011 .513$

\begin{abstract}
Recent studies have suggested that the RAS protein activator like-1 (RASAL1) is a potential tumor suppressor, which is found to be reduced in certain human cancers. Its downregulation is involved in the progression of malignancies. However, whether or not RASAL1 plays a role in the development of gastric cancer remains to be determined. Our study aimed to clarify the role of RASAL1 in the progression of gastric adenocarcinoma. The expression of RASAL1 in primary gastric adenocarcinoma tissue specimens was determined by immunohistochemistry. The expression of RASAL1 mRNA and protein was detected by RT-PCR and western blotting in gastric adenocarcinoma cell lines with varying differentiation statuses, including well-differentiated MKN-28, moderately differentiated SGC-7901 and poorly differentiated BGC-823, respectively. A normal gastric epithelial cell line, GES-1, was used as the control line. The immunohistochemical results revealed that the expression of the RASAL1 protein was mainly observed in the cytoplasm. Among 50 cases of gastric adenocarcinoma tissues, 12 cases were identified as (-), 23 cases $(+), 13$ cases $(++)$ and 2 cases $(+++)$. Among 50 cases of normal gastric tissues, 16 cases were $(++)$ and 34 cases $(+++)$. The expression of the RASAL1 protein was found to be decreased in the gastric adenocarcinoma tissue compared with normal gastric tissue $(\mathrm{p}<0.01)$. Moreover, in the gastric carcinoma tissues, the expression of RASAL1 was correlated with carcinoma diameter, differentiation grades, invasive depth, lymph node metastasis and TNM. Additionally, the RASAL1 mRNA and proteins were decreased in the three gastric adenocarcinoma cell lines compared with the normal gastric epithelial cell line GES-1. In addition, the downregulation of RASAL1 correlated with the differentiation status of cancer cell lines. Based on the above investigation, we conclude that expression of the
\end{abstract}

Correspondence to: Dr Hong Chen, Department of Gastroenterology, Zhongda Hospital, Southeast University, Nanjing 210009, Jiangsu, P.R. China

E-mail: njchenhong66@yahoo.com.cn

Key words: RAS protein activator like-1 gene, gastric adenocarcinoma
RASAL1 gene is decreased in gastric carcinoma tissues and cell lines. The results indicate that RASAL1 may be important in the tumorigenesis and development of gastric carcinoma.

\section{Introduction}

Gastric adenocarcinoma is one of the most frequent malignant tumors, with a high worldwide mortality. The clinical outcomes remain unsatisfactory, which is mainly due to a poor understanding of the mechanism of gastric cancer development and a lack of specific target gene therapy. To improve the survival rate, the development of novel treatments against molecular targets is crucial. Multiple genetic and epigenetic alterations are known to be involved in the tumorigenesis and progression of gastric cancer. Since Weinberg and Barbacid first cloned the C-Ha-Ras from human urinary bladder cancer in 1982, investigations have been conducted into the role of Ras in various malignant tumors (1-3). The Ras protein activator like-1 (RASAL1) gene was previously identified (4). The protein, encoded by the RASAL1 gene, is a member of the GTPase activating proteins (GAPs) family. It enhances the intrinsic GTPase activity of Ras proteins, resulting in the inactive GDP-bound form of Ras, thereby allowing control of cell proliferation and differentiation. The RASAL1 gene is reportedly correlated with the formation and development of colon cancer (5-6). In this study, we examined RASAL1 expression in vitro and in vivo and its clinicopathological significance in gastric adenocarcinoma.

\section{Materials and methods}

\section{Clinical cases}

Patients and clinical tissue specimens. A total of 50 patients diagnosed with primary gastric adenocarcinoma, who underwent surgically partial or total gastrectomy between August 2009 and March 2010 in the Affiliated Zhongda Hospital of the Southeast University (Nanjing, China), with available clinical information, were included in the study. No patients received chemotherapy or radiotherapy prior to surgery. The clinical stages and pathological features were defined according to the TNM Cancer Staging System of the American Joint Committee on Cancer. Paired primary gastric cancer and adjacent normal tissues were collected. The specimens were formalin-fixed, 
paraffin-embedded and cut into $4-\mu \mathrm{m}$ sections, which were stained with hematoxylin and eosin for histopathological type, differentiation stage and immunohistochemical evaluation. Written informed consent was obtained from all patients. The study was approved by the ethics committee of Zhongda Hospital, Southeast University.

Immunohistochemical analysis. Immunohistochemistry was used to detect the expression of RASAL1 in the specimens using a SP kit (Beijing Zhongshan Goldenbridge Biotechnology Company, China) according to the manufacturer's instructions. The working anti-human rabbit RASAL1 polyclonal antibody (Abcam, Cambridge, UK) was diluted at 1:200. The results were judged by two observers independently. RASAL1 expression was determined by assessing the percentage and intensity of stained tumor cells. The percentages of positive cells (percentage scores) were recorded as: $<5 \%$ (score 0 ), $6-25 \%$ (score 1 ), $26-50 \%$ (score 2 ) and $>51 \%$ (score 3). The staining intensities (intensity scores) were classified as: no staining (score 0 ), light brown staining (score 1), brown staining (score 2) and dark brown staining (score 3 ). RASAL1 staining positivity was calculated using the formula: overall score $=$ percentage score $\mathrm{x}$ intensity score. An overall score of $<1,2-3,4-6$ and $>6$ was defined as negative $(-)$, weak positive $(+)$, moderate positive $(++)$ and strong positive $(+++)$, respectively. For negative controls, sections were processed as above but treated with $0.01 \mathrm{~mol} / 1$ phosphate-buffered saline instead of primary antibodies.

\section{Experimental studies}

Cell lines. The well-differentiated gastric adenocarcinoma cell MKN-28, the moderately differentiated gastric adenocarcinoma cell SGC-7901 and the poorly differentiated gastric adenocarcinoma cell BGC-823 were obtained from the Shanghai Institute of Biochemistry and Cell Biology, China. The immortalized normal gastric epithelial cell line GES-1 was obtained from the Shanghai Institute of Digestive Disease, China. The cell lines were cultured and maintained in RPMI-1640 media and supplemented with $10 \%$ fetal bovine serum, penicillin and streptomycin in a humidified cell incubator with an atmosphere of $5 \% \mathrm{CO}_{2}$ at $37^{\circ} \mathrm{C}$.

Evaluation of RASAL1 $\mathrm{mRNA}$ in human gastric cancer cell lines. Total RNA was extracted from $2 \times 10^{5}$ cells (MKN-28, SGC-7901, BGC-823 and GES-1) by TRIzol reagent (Invitrogen, CA, USA). RNA $(1 \mu \mathrm{g})$ was converted into cDNA using the reverse transcription system with oligo-dT (Promega, Madison, WI, USA). PCR was carried out for RASAL1 using the primers: 5'-TGGATTTCTCTTCTTGCGATTCT-3' (forward) and 5'-TGTTGTCCCGAAGGTCAA-3' (reverse) (5). With $\beta$-actin acting as an internal control, the primers used were: 5'-TGCTATCCCTGTACGCCTCT-3' (forward) and 5'-AGTACTTGCGCTCAGGAGGA-3' (reverse) (7). The PCR conditions were $94^{\circ} \mathrm{C}$ for $2 \mathrm{~min}, 94^{\circ} \mathrm{C}$ for $30 \mathrm{sec}, 55^{\circ} \mathrm{C}$ for $30 \mathrm{sec}$ and $72^{\circ} \mathrm{C}$ for $1 \mathrm{~min}$ for 32 cycles. PCR products were separated by $2 \%$ agarose gel electrophoresis, stained with ethidium bromide (EB) and visualized using the ImageMaster VDS system (GE Healthcare, UK). Electrophoresis strips were analyzed by TotalLab 2.0 software (Nonlinear Dynamics Ltd). The ratio of the integrated density values (IDVs) for the RASAL1 transcripts to those for the $\beta$-actin transcripts was calculated. Subsequently, a comparison was made of the
RASAL1 mRNA levels for the gastric cancer and the normal gastric epithelial cell lines. The experiments were repeated three times to verify the results and the mean value for the RASAL1 mRNA expression was used for subsequent analysis.

Western blotting analysis. Western blotting analysis was performed to detect RASAL1 protein expression in the cell lines (MKN-28, SGC-7901, BGC-823 and GES-1) according to standard protocol. Cells were lysed in Mammalian Protein Extraction Reagent (M-PER) (Pierce, Rockford, IL, USA) containing a cocktail of proteinase inhibitors(Bio-Rad,Hercules, CA, USA). The lysed proteins were quantified by a bicinchoninic acid protein assay kit. Subsequently, equal amounts of proteins were electrophoresed on $10 \%$ SDS-polyacrylamide gels (SDS-PAGE) and then electroblotted onto nitrocellulose (Bio-Rad). The anti-human goat RASAL1 polyclonal antibody (Abcam) was used at a 1:200 dilution. The signal was visualized with an alkaline horseradish peroxidase-conjugated rabbit antigoat antibody (1:5000, Jingmei, Biotech, Beijing, China) and an enhanced chemiluminescence detection system (Amersham Pharmacia Biotech, Freiburg, Germany). The experiments were repeated three times to verify the results and the mean value was used for subsequent analysis.

Statistical analysis. Results were shown as the mean \pm standard deviation (SD). Statistical analysis was performed using SPSS 16.0 software. A Chi-square test, t-test and rank sum test were used. $\mathrm{P}<0.05$ was considered to indicate a statistically significant difference.

\section{Results}

\section{Clinical cases}

Expression patterns of RASAL1 by immunohistochemistry in gastric cancer tissues. The RASAL1 protein status of 50 gastric carcinoma and paired adjacent normal tissue samples were determined by immunohistochemical staining (Fig. 1, Table I). The expression of the RASAL1 protein was mainly observed in the cytoplasm. The results showed that the RASAL1 protein expression was (-) in 12 cases, (+) in 23 cases, (++) in 13 cases and $(+++)$ in 2 cases in gastric carcinoma tissues and $(++)$ in 16 cases and $(+++)$ in 34 cases in adjacent normal tissues. The results indicated that RASAL1 protein expression is significantly reduced in gastric carcinoma compared to adjacent normal tissues $(\mathrm{p}<0.05)$.

Clinicopathological characteristics of RASAL1 protein expression in gastric carcinoma. The correlation between clinicopathological characteristics of gastric carcinoma tissue samples and the RASAL1 protein expression was examined (Table II). This analysis revealed that the expression of the RASAL1 protein was decreased in gastric adenocarcinoma tissue compared to normal gastric tissue $(\mathrm{p}<0.01)$. In gastric carcinoma tissues, the reduced RASAL1 expression was associated with tumor size $(\mathrm{p}=0.001)$, differentiation degree $(\mathrm{p}=0.001)$, invasion depth $(\mathrm{p}=0.035)$ and lymph node metastasis $(\mathrm{p}=0.001)$, but not with age and gender.

\section{Experimental studies}

Expression of RASAL1 mRNA in human gastric cancer cell lines. The results of RT-PCR revealed that the expression of RASAL1 mRNA was decreased in the three tested 
Table I. RASAL1 protein in gastric carcinoma and normal tissues.

\begin{tabular}{|c|c|c|c|c|c|}
\hline \multirow[b]{2}{*}{ Tissue } & \multirow[b]{2}{*}{ No. of cases (n) } & \multicolumn{4}{|c|}{ RASAL1 expression n (\%) } \\
\hline & & - & + & ++ & +++ \\
\hline Carcinoma & 50 & $12(24.0)$ & $23(46.0)$ & $13(26.0)$ & $2(4.0)$ \\
\hline Normal & 50 & $0(0.0)$ & $0(0.0)$ & $16(32.0)$ & $34(68.0)$ \\
\hline
\end{tabular}

$\mathrm{P}<0.05$, gastric carcinoma tissues vs. normal gastric tissues; RASAL1, RAS protein activator like-1.
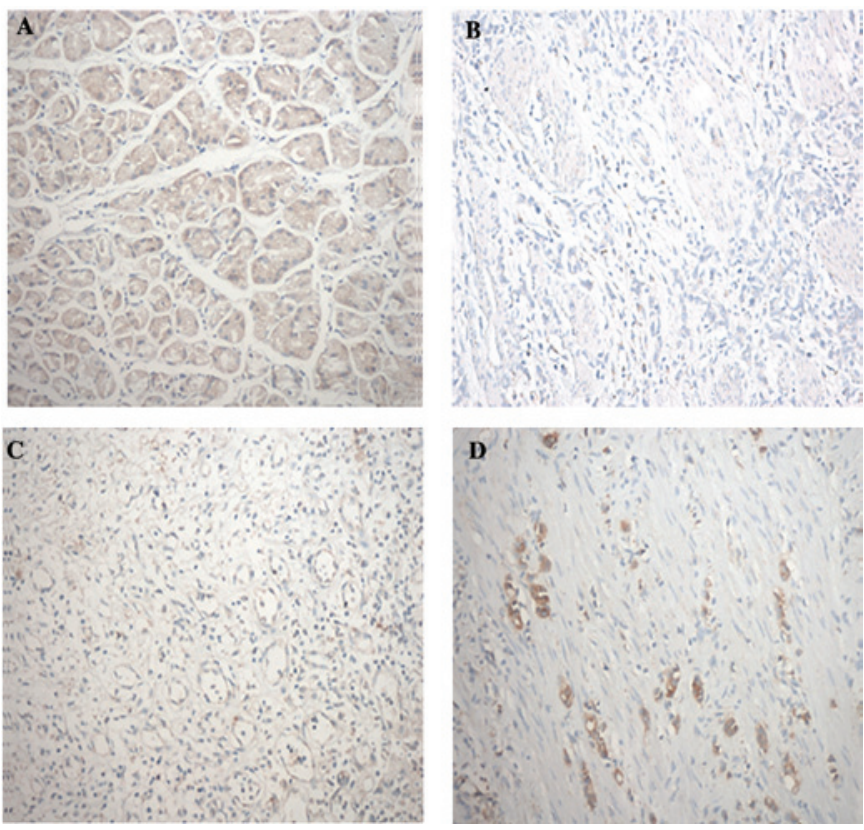

Figure 1. RASAL1 protein expression in normal gastric mucosa and gastric adenocarcinoma tissue was detected by immunohistochemistry (magnification, x100). (A) Normal gastric mucosa, RASAL1 (+++); (B) gastric adenocarcinoma tissue, RASAL1 (-); (C) gastric adenocarcinoma tissuse, RASAL1 (+); (D) gastric adenocarcinoma tissue, RASAL1 (++). RASAL1, RAS protein activator like-1.

gastric adenocarcinoma cell lines compared to the normal gastric epithelial cell line GES-l. The rates of down-regulated expression were $47.53 \%$ for MKN-28, 85.80\% for SGC-7901 and $95.86 \%$ for BGC-823 (Fig. 2, Table III). The expression level of RASAL1 mRNA correlated with the differentiation of the cells: cells with the poorest differentiation had the lowest expression. Among the three types of differentiated gastric cancer cells, the expression of RASAL1 mRNA was different $(\mathrm{p}<0.01)$.

Expression of RASAL1 protein in human gastric cancer cell lines. The results of western blotting revealed that the expression of RASAL1 protein was decreased in the three gastric cancer cell lines compared to the normal gastric epithelial cell line GES-1. The rates of downregulated expression were $40.12 \%$ for MKN-28, 54.94\% for SGC-7901 and 63.33\% for BGC-823 $(\mathrm{p}<0.01)$ (Fig. 3, Table IV). The expression levels of the RASAL1 protein was also correlated with differentiation: cells with the poorest differentiation had the lowest expression. Among the three types of differentiated gastric cancer cells, the expression of RASAL1 protein was different $(\mathrm{F}=3059.420, \mathrm{p}<0.01)$.

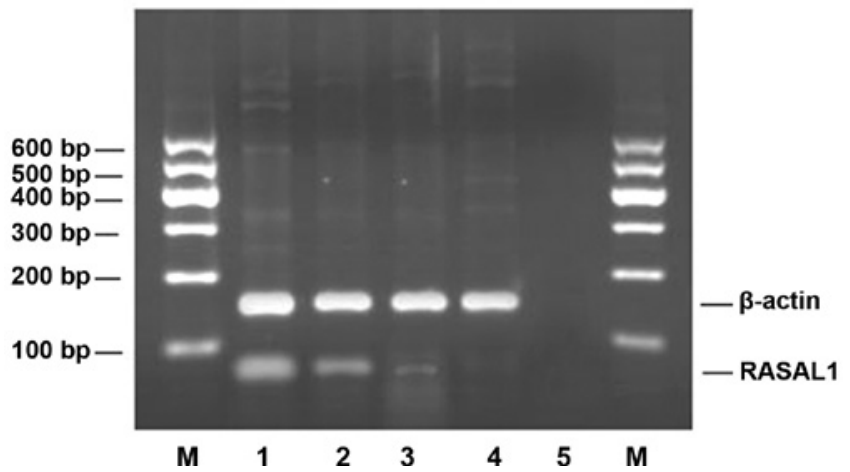

Figure 2. The expression of RASAL1 mRNA in human gastric adenocarcinoma cell lines. M, marker; lane 1, GES-1 cell line; lane 2, MKN-28 cell line; lane 3, SGC-7901 cell line; lane 4, BGC-823 cell line and lane; 5, negative control. RASAL1, RAS protein activator like-1.

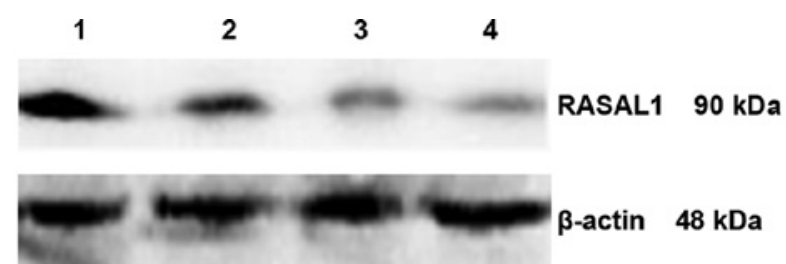

Figure 3. The expression of RASAL1 protein in human gastric cancer cell lines. Lane 1, GES-1 cell line; lane 2, MKN-28 cell line; lane 3, SGC-7901 cell line; lane 4, BGC-823 cell line. RASAL1, RAS protein activator like-1.

\section{Discussion}

The Ras family comprises three members including K-Ras, $\mathrm{H}-\mathrm{R}$ as and N-Ras that encode proteins known as p21, which play a key role in transducing growth signals from the cell surface to the cell nucleus. The activation of Ras signaling, such as the 'Ras-RAF-MEK-ERK' signaling cascade, causes cell proliferation, differentiation and survival. The role of the Ras gene in the development of malignant tumors has always been a focus of research. Currently, it is known that the aberrant activation of Ras signaling causes persistent cell proliferation and leads to tumorigenesis (8).

Ras genes are well investigated due to their frequent activation and mutation in human cancers. Abnormal Ras signaling activation is mainly caused by mutations. Ras mutations lead to constantly activated Ras signaling in the absence of extracellular signals. Activating mutations of Ras are found in 
Table II. Correlation between RASAL1 protein and clinicopathological characteristics in gastric carcinoma.

\begin{tabular}{|c|c|c|c|c|c|c|}
\hline \multirow[b]{2}{*}{ Group } & \multirow[b]{2}{*}{ No. of cases (n) } & \multicolumn{4}{|c|}{ RASAL1 expression (n) } & \multirow[b]{2}{*}{$\mathrm{p}$-value } \\
\hline & & - & + & ++ & +++ & \\
\hline Gender & & & & & & 0.370 \\
\hline Male & 34 & 8 & 17 & 9 & 0 & \\
\hline Female & 16 & 4 & 6 & 4 & 2 & \\
\hline Age $(y r)$ & & & & & & 0.554 \\
\hline$<60$ & 19 & 5 & 7 & 6 & 1 & \\
\hline$\geq 60$ & 31 & 7 & 16 & 7 & 1 & \\
\hline Tumor size (cm) & & & & & & 0.001 \\
\hline$<4$ & 22 & 1 & 10 & 9 & 2 & \\
\hline$\geq 4$ & 28 & 11 & 13 & 4 & 0 & \\
\hline Differentiation & & & & & & 0.001 \\
\hline Well, moderately & 21 & 0 & 8 & 11 & 2 & \\
\hline Poorly & 29 & 12 & 15 & 2 & 0 & \\
\hline Invasion depth ${ }^{\mathrm{a}}$ & & & & & & 0.035 \\
\hline $\mathrm{m}, \mathrm{sm}$ & 10 & 1 & 4 & 3 & 2 & \\
\hline mp or deeper & 40 & 11 & 19 & 10 & 0 & \\
\hline Lymph node metastasis & & & & & & 0.001 \\
\hline Negative & 13 & 0 & 4 & 8 & 1 & \\
\hline Positive & 37 & 12 & 19 & 5 & 1 & \\
\hline TNM stage & & & & & & 0.034 \\
\hline $1-2$ & 16 & 1 & 5 & 10 & 0 & \\
\hline $3-4$ & 34 & 11 & 18 & 3 & 2 & \\
\hline
\end{tabular}

anvasion depth: m, mucosa; sm, submucosa; mp, muscularis propria. RASAL1, RAS protein activator like-1.

Table III. Expression of RASAL1 mRNA in human gastric adenocarcinoma cell lines.

\begin{tabular}{lcc}
\hline Group & RASAL1 $/ \beta$-actin $($ mean \pm SD) & Rate of downregulated expression $(\%)$ \\
\hline GES-1 & $0.507 \pm 0.005$ & - \\
MKN-28 & $0.266 \pm 0.009$ & $47.53^{\mathrm{a}}$ \\
SGC-7901 & $0.072 \pm 0.004$ & $85.80^{\mathrm{a}}$ \\
BGC-823 & $0.021 \pm 0.003$ & $95.86^{\mathrm{a}}$ \\
\hline
\end{tabular}

${ }^{a} \mathrm{P}<0.01$, compared with GES-1. RASAL1, RAS protein activator like-1.

Table IV. Expression of RASAL1 protein in human gastric cancer cell lines.

\begin{tabular}{lcc}
\hline Group & RASAL1/ $\beta$-actin (mean \pm SD) & Rate of downregulated expression $(\%)$ \\
\hline GES-1 & $0.810 \pm 0.008$ & - \\
MKN-28 & $0.485 \pm 0.009$ & $40.12^{\mathrm{a}}$ \\
SGC-7901 & $0.365 \pm 0.013$ & $54.94^{\mathrm{a}}$ \\
BGC-823 & $0.297 \pm 0.005$ & $63.33^{\mathrm{a}}$ \\
\hline
\end{tabular}

${ }^{a} \mathrm{P}<0.01$, compared with GES-1. RASAL1, RAS protein activator like-1. 
$20-25 \%$ of all human tumors and in up to $90 \%$ of certain tumor types. While mutations of H-Ras and N-Ras do not frequently occur, high levels of K-Ras mutations are found in leukemias, colon, pancreatic and lung cancer (9). However, in the absence of a mutation, it is difficult to explain the observed high Ras signaling. For example, in some tumor entities, including in gastric cancer, although Ras mutations are seldom detected, Ras activity is still unusually high (10-11). The underlying mechanisms regarding this remain largely unknown.

Findings of other studies have shown a novel mechanism for Ras activity regulation besides point mutation (4-6). Ras has two structural conformations, 'on' and 'off'. When Ras is bound to the nucleotide guanosine diphosphate (GDP), it is in an 'off' state, whereas Ras bound to guanosine triphosphate (GTP) is in an 'on' state. The activation and deactivation of Ras is controlled by GAPs and guanine nucleotide exchange factors (GEFs) (12). The GAPs have GTP enzyme activity, which facilitate converting Ras from an active GTP-bound state to an inactive GDP-bound state. Inversely, the GEFs change Ras from an inactive GDP-bound state to an active GTP-bound state. The balance between GEF and GAP activity determines the guanine nucleotide status of Ras, thereby regulating Ras activity. Since Ras GAPs switch off Ras signaling transduction pathways, the genes encoding these RAS GAPs are considered to be potential tumor suppressor genes. A number of studies have indicated that certain members of the Ras GAP family are indeed tumor suppressors, including neurofibromin (NF1) and DAB2IP (13-17).

The RASAL1 gene, which is located on chromosome 12 (12q23-q24), has an overall length of $1100 \mathrm{bp}$ and a structure mainly consisting of exon 1 and 2 and $\mathrm{Ca}^{2+}$-binding sites. As a newly discovered gene, it was proved to regulate the activity of the Ras signal transduction pathway (4). The RASAL1 gene possesses the characteristics of Ras GAPs, which enhance the intrinsic Ras-GTPase activity through hydrolyzing GTP into GDP, and thus is involved in cell differentiation, generation and apoptosis. Jin et al found that RASAL1 is expressed in almost all normal tissues, including in the heart, kidney, gastrointestinal tract, pancreas, lung, prostate and bone marrow, suggesting that RASAL1 genes possess a significant role in maintaining various normal human physiological functions (4). In recent studies, RASAL1 was found to be downregulated in a variety of tumor tissues, including nasopharyngeal, breast, lung, liver and esophageal cancer and lymphoma. By studying 152 patient colorectal cancer tissues and 18 types of colon cancer cells, Ohta et al found that the expression of RASAL1 decreased markedly in colorectal cancer cells that contained the wild-type $K$-Ras gene, but did not decrease in those colorectal cancer cells with a mutant $K$-Ras gene (5). RASAL1 expression was detected in $46.9 \%$ $(30 / 64)$ of adenocarcinoma, $17.4 \%(8 / 46)$ of large adenoma and none of the small adenoma samples $(0 / 42)$. Based on the above study, RASAL1 was found to inhibit tumor progression by downregulating the Ras signal activity. It was also found that the ectopic expression of RASAL1 in transfected colorectal cancer cells in culture promoted Ras inactivation, which was confirmed by the depression of ERK, the downstream effector in the Ras signaling transduction pathway, as well as the suppression of the malignant phenotype in colorectal cancer cells. In their study, Calvisi et al also found that in the absence of Ras mutations, the downregulation of RASAL1, as well as other Ras GAPs (DAB2IP and NF1), resulted in the unrestrained activation of Ras signaling in the presence of wild-type Ras in human hepatocarcinogenesis (12), but it is unknown whether or not this also occurs in gastric cancer.

In our study, using immunohistochemistry, it was found that of 50 cases of gastric cancer tissues 12 cases were (-), 23 cases $(+), 13$ cases $(++)$ and 2 cases $(+++)$; whereas in 50 cases of normal gastric tissues 16 cases were $(++)$ and 34 cases were $(+++)$. This finding indicates that the RASAL1 expression is significantly reduced in gastric cancer tissues as well. Furthermore, we found that a reduced RASAL1 expression was associated with tumor size, differentiation degree, invasion depth and lymph node metastasis, but not with age and gender. RT-PCR and western blotting were used to detect the expression of RASAL1 in three types of gastric adenocarcinoma cell lines, including well-, moderately and poorly differentiated adenocarcinoma cells. The results confirmed that RASAL1 expression decreased in the three types of gastric adenocarcinoma cells compared with the normal gastric epithelial cell line, and the expression level was correlated with the differentiation: the poorest differentiation had the lowest expression. In a recent study, Seto et al investigated 10 gastric cancer cell lines by immunoblotting, and found that RASAL1 expression was reduced in 6 out of the 10 cell lines (18). These authors also reported that the immunohistochemical analyses in primary gastric tumors revealed that the RASAL1 expression was reduced in 23 out of 48 (48\%) of the gastric cancers cell lines, but in none of the adenomas $(0 / 10)$. These results suggest that RASAL1 is important in the tumorigenesis and development of gastric carcinoma. The results of our study have shed light on the pathogenesis of gastric carcinoma, and are a new therapeutic target for gastric carcinoma treatment.

Epigenetic silencing has been found to be the key mechanism responsible for the downregulated expression of the RASAL1 gene in tumors. Evidence indicates that $\mathrm{CpG}$ island methylation in the promoter of the RASAL1 gene may induce silencing in multiple tumors, including in esophageal cancer, nasopharyngeal carcinoma and colorectal cancer cells (4). Simultaneously, other studies have demonstrated that in colon cancer cells, the gene methylation of RASAL1 was reversed by using the methylation inhibitor 5-acetazolamide-2-cytosine deoxyriboside (5-Aza-CdR) (19). Kolfschoten et al, however, found another important mechanism affecting RASAL1 expression. Since RASAL1 is a transcription target of the antioncogene pituitary homeobox 1 (PITX1), RASAL1 expression may be downregulated by a decrease in PITX1 gene expression in human gastric carcinogenesis (20-21).

In conclusion, our findings demonstrate that the expression of RASAL1 is markedly decreased in gastric carcinoma tissues and cell lines, and is associated with gastric carcinoma differentiation degree and progression, suggesting that it is important in the development of gastric carcinoma. The mechanisms that contribute to the downregulated expression of RASAL1 in gastric cancer require further investigation.

\section{Acknowledgements}

This study was supported by the Natural Science Foundation of Jiangsu Province of China (No. BK2008301). 


\section{References}

1. Parada LF, Tabin CJ, Shis C and Weinberg RA: Human EJ bladder carcinoma oncogene is homologue of Harvey sarcoma virus ras gene. Nature 297: 474-478, 1982.

2. Fernández-Medarde A and Santos E: Ras in cancer and developmental diseases. Genes Cancer 2: 344-358, 2011.

3. Cox AD and Der CJ: Ras history: the saga continues. Small Gtpases 1: 2-27, 2010.

4. Jin $\mathrm{H}$, Wang X, Ying J, Wong AH, Cui Y, Srivastava G, Shen ZY, Li EM, Zhang Q, Jin J, Kupzig S, Chan AT, Cullen PJ and Tao Q: Epigenetic silencing of $\mathrm{Ca}^{2+}$-regulated Ras GTPase-activating protein RASAL defines a new mechanism of Ras activation in human cancers. Proc Natl Acad Sci USA 104: 12353-12358, 2007.

5. Ohta M, Seto M, Ijichi H, Miyabayashi K, Kudo Y, Mohri D, Asaoka Y, Tada M, Tanaka Y, Ikenoue T, Kanai F, Kawabe T and Omata M: Decreased expression of the Ras GTPase-activating protein RASAL1 is associated with colorectal tumor progression. Gastroenterology 136: 206-216, 2009.

6. Bernards A and Settleman J: Loss of the Ras regulator RASAL1: another route to Ras activation in colorectal cancer. Gastroenterology 136: 46-48, 2009.

7. Wang YW, Qu Y, Li JF, Chen XH, Liu BY, Gu QL and Zhu ZG: In vitro and in vivo evidence of metallopanstimulin-1 in gastric cancer progression and tumorigenicity. Clin Cancer Res 12: 4965-4973, 2006.

8. Malumbres $\mathrm{M}$ and Barbacid M: RAS oncogenes: the first 30 years. Nat Rev Cancer 3: 459-465, 2003.

9. Karnoub AE and Weinberg RA: Ras oncogenes: split personalities. Nat Rev Mol Cell Biol 9: 517-531, 2008.

10. Kimura K, Nagasaka T, Hoshizima N, Sasamoto H, Notohara K, Takeda M, Kominami K, Iishii T, Tanaka N and Matsubara N: No duplicate KRAS mutation is identified on the same allele in gastric or colorectal cancer cells with multiple KRAS mutations. J Int Med Res 35: 450-457, 2007.

11. Liu ZM, Liu LN, Li M, Zhang QP, Cheng SH and Lu S: Mutation detection of KRAS by high-resolution melting analysis in Chinese with gastric cancer. Oncol Rep 22: 515-520, 2009.

12. Calvisi DF, Ladu S, Conner EA, Seo D, Hsieh JT, Factor VM and Thorgeirsson SS: Inactivation of Ras GTPase-activating proteins promotes unrestrained activity of wild-type Ras in human liver cancer. J Hepatol 54: 311-319, 2011.
13. Kong Z, Xie D, Boike T, Raghavan P, Burma S, Chen DJ, Habib AA, Chakraborty A, Hsieh JT and Saha D: Downregulation of human DAB2IP gene expression in prostate cancer cells results in resistance to ionizing radiation. Cancer Res 70: 2829-2839, 2010.

14. Vigil D, Cherfils J, Rossman KL and Der CJ: Ras superfamily GEFs and GAPs: validated and tractable targets for cancer therapy? Nat Rev Cancer 10: 842-857, 2010.

15. Bos JL, Rehmann $\mathrm{H}$ and Wittinghofer A: GEFs and GAPs: critical elements in the control of small $G$ proteins. Cell 129 : 865-877, 2007.

16. Iwashita S and Song SY: Ras GAPs: a crucial regulator of extracellular stimuli for homeostasis of cellular functions. Mol Biosyst 4: 213-222, 2008.

17. Hölzel M, Huang S, Koster J, Ora I, Lakeman A, Caron H, Nijkamp W, Xie J, Callens T, Asgharzadeh S, Seeger RC, Messiaen L, Versteeg R and Bernards R: NF1 is a tumor suppressor in neuroblastoma that determines retinoic acid response and disease outcome. Cell 142: 218-229, 2010.

18. Seto M, Ohta M, Ikenoue T, Sugimoto T, Asaoka Y, Tada M, Mohri D, Kudo Y, Ijichi H, Tateishi K, Otsuka M, Hirata Y, Maeda S, Koike K and Omata M: Reduced expression of RAS protein activator like-1 in gastric cancer. Int J Cancer 128: 1293-1302, 2011

19. Liu Q, Walker SA, Gao D, Taylor JA, Dai YF, Arkell RS, Bootman MD, Roderick HL, Cullen PJ and Lockyer PJ: CAPRI and RASAL impose different modes of information processing on RAS due to contrasting temporal filtering of $\mathrm{Ca}^{2+}$. J Cell Biol 170: 183-190, 2005.

20. Kolfschoten IG, van Leeuwen B, Berns K, Mullenders J, Beijersbergen RL, Bernards R, Voorhoeve PM and Agami R: A genetic screen identifies PITX1 as a suppressor of RAS activity and tumorigenicity. Cell 121: 849-858, 2005.

21. Chen YN, Chen H, Xu Y, Zhang X and Luo Y: Expression of pituitary homeobox 1 gene in human gastric carcinogenesis and its clinicopathological significance. World J Gastroenterol 14: 292-297, 2008. 